\title{
Sensitivity of strange quark polarization to flavor $S U(3)$ symmetry breaking
}

\author{
Ali Khorramian $\odot^{*}$ \\ Faculty of Physics, Semnan University, 35131-19111 Semnan, Iran \\ Elliot Leader $\odot^{\dagger}$ \\ Imperial College, Prince Consort Road, London SW7 2BW, England \\ Dimiter B. Stamenov $\odot^{\ddagger}$ \\ Institute for Nuclear Research and Nuclear Energy, Bulgarian Academy of Sciences, \\ Boulevard Tsarigradsko Chaussee 72, Sofia 1784, Bulgaria \\ Amir Shabanpour $\oplus^{\S}$ \\ Faculty of Physics, Semnan University, 35131-19111 Semnan, Iran
}

(Received 11 September 2020; accepted 29 January 2021; published 2 March 2021)

\begin{abstract}
The polarized strange quark puzzle concerns the fact that the polarized strange quark density extracted from polarized inclusive deep inelastic scattering data is significantly negative, whereas it is zero or slightly positive when extracted from a combined analysis of polarized semi-inclusive and inclusive deep inelastic data. $S U(3)$ flavor symmetry, which, it is generally accepted, is not an exact symmetry, plays an important role in the inclusive analysis, and all the extracted polarized quark densities depend, to some extent, on the level of symmetry breaking introduced. But by far the most sensitive to the breaking is the strange quark density. In this paper we present a NLO QCD analysis of the world data on polarized inclusive DIS data on protons, neutrons and deuterons, including the final JLAB CLAS/EG1b data on the proton and deuteron, and study the sensitivity of the strange quark polarization to the breaking of flavor $S U(3)$.
\end{abstract}

DOI: 10.1103/PhysRevD.103.054003

\section{INTRODUCTION}

Our knowledge of the internal partonic spin structure of the nucleon comes mainly from the polarized inclusive and semi-inclusive deep inelastic scattering (DIS) of leptons on nucleons. While the inclusive DIS processes, in the lack of neutrino reactions on a polarized target at present, determine only the sum of quark and antiquark polarized parton density functions (PPDFs), $(\Delta q(x)+\Delta \bar{q}(x))$, the polarized semi-inclusive DIS data could provide information about the individual polarized quark and antiquark densities, if the fragmentation functions (FFs) are well determined.

All QCD analyses of the polarized inclusive DIS data, in which the flavor $S U(3)$ symmetry is not broken, have produced negative values for the polarized strange quark

\footnotetext{
*Khorramiana@semnan.ac.ir

†.leader@imperial.ac.uk

tstamenov@inrne.bas.bg

§amir.shabanpourph@semnan.ac.ir
}

Published by the American Physical Society under the terms of the Creative Commons Attribution 4.0 International license. Further distribution of this work must maintain attribution to the author(s) and the published article's title, journal citation, and DOI. Funded by SCOAP ${ }^{3}$. density, $\left(\Delta s\left(x, Q^{2}\right)+\Delta \bar{s}\left(x, Q^{2}\right)\right)$ (see for instance ([1-6]) ). Note that in the majority of these analyses simple input parametrizations for the polarized strange quark density, which do not permit a sign change of the density, have been used.

It was shown [6], however, that even allowing in the parametrization of the polarized strange quark density for a possible sign change, in the presence of the then available more precise data, the inclusive analysis still yielded significantly negative values for the polarized strange quark density, except for negligible positive values in the region $x>0.3$.

On the other hand, the strange quark density obtained from combined QCD analyses of inclusive and semiinclusive polarized deep inelastic scattering data ([7-9]) turns out to be positive in the $x$ range $0.02<x<0.2$ and negative for $x<0.02$. This disagreement between the inclusive and semi-inclusive analyses is known as the strange quark polarization puzzle.

It was shown [10] and understood that in the presence of the polarized semi-inclusive data the polarized strange quark density is very sensitive to the kaon fragmentation functions used in the analysis, and more generally, that to obtain correct values for the polarized individual quark and antiquark densities it is crucial that the fragmentation functions to be reliably determined. 
It is important to mention that in the QCD analyses of the polarized inclusive DIS data alone, the $S U(3)$ symmetric value $3 \mathrm{~F}-\mathrm{D}$ for the nonsinglet axial charge $a_{8}$ (with parameters $\mathrm{F}$ and $\mathrm{D}$ determined from the hyperon $\beta$ decays analysis, see for instance [11]) is usually used, but it is of interest to know to what extent the $S U(3)$ symmetry is believed to be broken. There is a growing precision of the measurements of magnetic moments, $g_{A} / g_{V}$ ratios and rates in hyperon $\beta$ decays, and different theoretical models ([12] and more recently [13]) have been used to study how large any flavor $S U(3)$ symmetry breaking should be in order to describe well these new data, and consequently how big could be the deviation of $a_{8}$ from its $S U(3)$ symmetric value. In this connection we would like to mention the QCD analysis [14] of the polarized inclusive DIS data, where for the uncertainty of the $S U(3)$ symmetry value of $a_{8}$ has been used a value approximately six times bigger than that obtained from the phenomenological analysis of the data on hyperon $\beta$ decays. This choice of the uncertainty actually means that the SU(3) symmetry is up to $30 \%$ broken.

In 2000 the first NLO QCD analysis [15] of the polarized inclusive DIS data was performed in order specifically to study the sensitivity of the polarized PDFs and their first moments to the $S U(3)$ symmetry breaking effects which were taken into account in model independent way. The main result was that most sensitive to the change of the value of $a_{8}$ is the polarized strange quark density, but it remains negative for all $x$.

In this paper we present a NLO QCD analysis of the world data set on the nucleon spin structure functions $g_{1}^{N}$ $(N=p, n, d)$ including the final JLAB CLAS/EG1b data on the proton [16] and deuteron [17] spin structure functions. The aim of our analysis is to further study the sensitivity of the polarized PDFs, and especially the polarized strange quark density, to the change of the value of the nonsinglet axial charge $a_{8}$ due to $S U(3)$ symmetric breaking effects, now that we have much more data and with higher accuracy and wider kinematic range than those available in 2000.

Unlike the QCD analyses of the polarized inclusive DIS data usually performed, the following changes are made:

(i) We use now input parametrizations for the sum of quark and antiquark polarized PDFs $\Delta q(x)+\Delta \bar{q}(x)$ instead of the valence and sea quarks densities, because as was stressed above only the sums $\Delta q\left(x, Q^{2}\right)+\Delta \bar{q}\left(x, Q^{2}\right)$ can be extracted from the data.

(ii) We do not make any assumptions about the polarized light sea quark densities $\Delta \bar{u}(x)$ and $\Delta \bar{d}(x)$ which have been used in almost all previous analyses, because such assumptions cannot be directly tested. Note here that in contrast to the light sea quark densities, the total strange quark density $(\Delta s+\Delta \bar{s})\left(x, Q^{2}\right)$ can be well determined from the inclusive data if they are sufficiently precise [18].

\section{QCD FRAMEWORK FOR INCLUSIVE POLARIZED DIS}

One of the features of polarized DIS is that more than half of the present data are at moderate $Q^{2}$ and hadronic final state mass squared $W^{2}\left(Q^{2} \sim 1-5 \mathrm{GeV}^{2}, 4 \mathrm{GeV}^{2}<\mathrm{W}^{2}<10 \mathrm{GeV}^{2}\right)$, or in the so-called preasymptotic region. This is especially the case for the very precise experiments performed at the Jefferson Laboratory. So, in contrast to the unpolarized case this region cannot be excluded from the analysis. As was shown in [1], to confront correctly the QCD predictions to the experimental data including the preasymptotic region, the nonperturbative higher twist (powers in $1 / Q^{2}$ ) corrections to the nucleon spin structure functions have to be taken into account too.

In QCD the spin structure function $g_{1}$ has the following form for $Q^{2} \gg \Lambda_{\mathrm{QCD}}^{2}$ (the nucleon target label " $N$ " is not shown):

$$
g_{1}\left(x, Q^{2}\right)=g_{1}\left(x, Q^{2}\right)_{\mathrm{LT}}+g_{1}\left(x, Q^{2}\right)_{\mathrm{HT}},
$$

where "LT" denotes the leading twist $(\tau=2)$ contribution to $g_{1}$, while "HT" denotes the contribution to $g_{1}$ arising from QCD operators of higher twist, namely $\tau \geq 3$.

$$
\begin{aligned}
g_{1}\left(x, Q^{2}\right)_{\mathrm{LT}}= & g_{1}\left(x, Q^{2}\right)_{\mathrm{pQCD}}+h^{\mathrm{TMC}}\left(x, Q^{2}\right) / Q^{2} \\
& +\mathcal{O}\left(M^{4} / Q^{4}\right),
\end{aligned}
$$

where $g_{1}\left(x, Q^{2}\right)_{\mathrm{pQCD}}$ is the well known (logarithmic in $Q^{2}$ ) NLO pQCD contribution

$$
\begin{aligned}
g_{1}\left(x, Q^{2}\right)_{\mathrm{pQCD}}= & \frac{1}{2} \sum_{q}^{n_{f}} e_{q}^{2}\left[(\Delta q+\Delta \bar{q}) \otimes\left(1+\frac{\alpha_{s}\left(Q^{2}\right)}{2 \pi} \delta C_{q}\right)\right. \\
& \left.+\frac{\alpha_{s}\left(Q^{2}\right)}{2 \pi} \Delta G \otimes \frac{\delta C_{G}}{n_{f}}\right]
\end{aligned}
$$

and $h^{\mathrm{TMC}}\left(x, Q^{2}\right)$ are the exactly calculable kinematic target mass corrections [19], which, being purely kinematic, effectively belong to the LT term. In Eq. (3), $\Delta q\left(x, Q^{2}\right), \Delta \bar{q}\left(x, Q^{2}\right)$ and $\Delta G\left(x, Q^{2}\right)$ are quark, anti-quark and gluon polarized densities in the proton, which evolve in $Q^{2}$ according to the spin-dependent NLO DGLAP equations. $\delta C(x)_{q, G}$ are the NLO spin-dependent Wilson coefficient functions calculated in $\overline{\mathrm{MS}}$ scheme and the symbol $\otimes$ denotes the usual convolution in Bjorken $x$ space. $n_{f}$ is the number of active flavors ( $n_{f}=3$ in our analysis).

In addition to the LT contribution, the dynamical higher twist effects 


$$
g_{1}\left(x, Q^{2}\right)_{\mathrm{HT}}=h\left(x, Q^{2}\right) / Q^{2}+\mathcal{O}\left(\Lambda^{4} / Q^{4}\right),
$$

must be taken into account at low $Q^{2}$. The latter are nonperturbative effects and cannot be calculated in a model independent way. That is why we prefer to extract them directly from the experimental data. Note also, that in our analysis the logarithmic $Q^{2}$ dependence of $h\left(x, Q^{2}\right)$ in Eq. (4), which is not known in QCD, is neglected. Compared to the principal $1 / Q^{2}$ dependence it is expected to be small and the accuracy of the present data does not allow its determination. Therefore, the extracted from the data values of the parameters $h^{N}\left(x_{i}\right)(N=p, n ; i=1,2, . .5)$ correspond to the mean $Q^{2}$ for each $x_{i}$-bin (see Fig. 6 and the discussion there).

In our analysis of the inclusive DIS data the inverse Mellin transformation method has been used to calculate the spin structure function $g_{1}\left(x, Q^{2}\right)_{\mathrm{LT}}$ from its moments taking into account the first order in $\mathcal{O}\left(M^{2} / Q^{2}\right)$ TMC. For the numerical calculations the Pegasus routines [20] have been used.

As was mentioned in the Introduction, we are using now input parametrizations at $Q_{0}^{2}=1 \mathrm{GeV}^{2}$ for the sum of quark and anti-quark polarized parton densities instead of the valence and sea quarks densities:

$$
\begin{aligned}
x(\Delta u+\Delta \bar{u})\left(x, Q_{0}^{2}\right) & =A_{u_{+}} x^{\alpha_{u_{+}}}(1-x)^{\beta_{u_{+}}}\left(1+\epsilon_{u_{+}} \sqrt{x}+\gamma_{u_{+}} x\right), \\
x(\Delta d+\Delta \bar{d})\left(x, Q_{0}^{2}\right) & =A_{d_{+}} x^{\alpha_{d_{+}}}(1-x)^{\beta_{d_{+}}}\left(1+\gamma_{d_{+}} x\right), \\
x(\Delta s+\Delta \bar{s})\left(x, Q_{0}^{2}\right) & =A_{s_{+}} x^{\alpha_{s_{+}}}(1-x)^{\beta_{s_{+}}}\left(1+\gamma_{s_{+}} x\right), \\
x \Delta G\left(x, Q_{0}^{2}\right) & =A_{G} x^{\alpha_{G}}(1-x)^{\beta_{G}}\left(1+\gamma_{G} x\right),
\end{aligned}
$$

and do not use any assumptions about the light sea quark densities $\Delta \bar{u}$ and $\Delta \bar{d}$. In (5) the notation $q_{+}=q+\bar{q}$ is used for $q=u, d, s$.

Usually the set of free parameters in (5) is reduced by the well known sum rules

$$
\begin{gathered}
a_{3}=g_{A}=\mathrm{F}+\mathrm{D}=1.270 \pm 0.003 \\
a_{8}=3 \mathrm{~F}-\mathrm{D}=0.586 \pm 0.031
\end{gathered}
$$

where $a_{3}$ and $a_{8}$ are non-singlet combinations of the first moments of the polarized parton densities corresponding to $3^{\text {rd }}$ and $8^{\text {th }}$ components of the axial vector Cabibbo current

$$
\begin{aligned}
a_{3}= & (\Delta u+\Delta \bar{u})\left(Q^{2}\right)-(\Delta d+\Delta \bar{d})\left(Q^{2}\right), \\
a_{8}= & (\Delta u+\Delta \bar{u})\left(Q^{2}\right)+(\Delta d+\Delta \bar{d})\left(Q^{2}\right) \\
& -2(\Delta s+\Delta \bar{s})\left(Q^{2}\right),
\end{aligned}
$$

and the values of parameters $\mathrm{F}$ and $\mathrm{D}$ are determined from the $S U(3)$ flavor symmetry analysis of the hyperon $\beta$ decays, and slightly change over the years due to the improvement of the precision of the experiments. The experimental values for $g_{A}$ in (6) and $a_{8}$ in (7), used in our study, are presented in [21] and [22], respectively.

The sum rule (6) reflects isospin $S U(2)$ symmetry, whereas (7) is a consequence of the $S U(3)_{f}$ flavor symmetry treatment of the hyperon $\beta$-decays. So, using the constraints (6) and (7) the parameters $A_{u+\bar{u}}$ and $A_{d+\bar{d}}$ in (5) are determined as functions of the parameters connected with $(\Delta u+\Delta \bar{u}),(\Delta d+\Delta \bar{d})$ and $(\Delta s+\Delta \bar{s})$.

The large $x$ behavior of the polarized PDFs is mainly controlled by the positivity constraints [8]. The only difference is that now we are using for the unpolarized NLO PDFs the MMHT'14 set of parton densities [23] instead the MRST'02 one. We have found that the positivity condition for the polarized strange quarks and gluons is guaranteed, if for the values of the parameters $\beta_{s+\bar{s}}$ and $\beta_{G}$, which control their large $x$ behavior, the values 9.0 and 5.0 are used, respectively.

The rest of the parameters $\left\{A_{i}, \alpha_{i}, \beta_{i}, \epsilon_{i}, \gamma_{i}\right\}$, as well as the unknown higher twist corrections $h^{N}\left(x_{i}\right) / Q^{2}$ to the spin structure functions $g_{1}^{N}\left(x, Q^{2}\right),(N=p, n)$ have been determined simultaneously from the best fit to the DIS data. Note that the $\sqrt{x}$ term has been used only in the parametrization for the $(\Delta u+\Delta \bar{u})$ density, because the parameters $\epsilon_{i}$ in front of it for the other polarized densities can not be determined from the fit, and do not help to improve it. The parameter $\gamma_{G}$ was fixed to zero because the accuracy of the present data do not also allow its determination. Concerning the parameter $\gamma_{s_{+}}$see the discussion below.

The method used to extract simultaneously the polarized parton densities and higher twist corrections from the data is described in [24].

In polarized DIS the $Q^{2}$ range and the accuracy of the data are much smaller than that in the unpolarized case. That is why, in all calculations we have kept fixed the value of the strong coupling constant $\alpha_{s}\left(Q_{0}^{2}\right)$ at the initial scale $Q_{0}^{2}=1 \mathrm{GeV}^{2}$. Thus, given the value of $\alpha_{s}\left(Q_{0}^{2}\right)$ we have numerically solved the differential equation for $\alpha_{s}\left(Q^{2}\right)$ for any $Q^{2}$ [20]. For $\alpha_{s}\left(Q_{0}^{2}\right)$ we have used the value 0.48780 obtained by the MMHT'14 NLO QCD analysis [23] of the world unpolarized data, which corresponds to $\alpha_{s}\left(M_{z}^{2}\right)=$ 0.120 . This value was chosen in order that the $Q^{2}$ evolution of the polarized PDFs would be consistent with the evolution of the unpolarized MMHT'14 PDFs which are used in the positivity constraints.

\section{RESULTS OF ANALYSIS}

In this section we will present and discuss the results of our new NLO QCD fit to the present world data on polarized inclusive DIS ([16,17,22,25-36]). The data used (682 experimental points) cover the following kinematic region: $\left\{0.004 \leq x \leq 0.75,1<Q^{2} \leq 96.1 \mathrm{GeV}^{2}\right\}$. Note that for the CLAS/EG1b data a cut $W>2 \mathrm{GeV}$ was imposed in order to exclude the resonance region. 
TABLE I. Data used in our NLO QCD analysis, the individual $\chi^{2}$ for each set and the total $\chi^{2}$ of the Fit A.

\begin{tabular}{llrr}
\hline \hline Experiment & Process & $N_{\text {data }}$ & \multicolumn{1}{c}{$\chi^{2}$} \\
\hline EMC [25] & DIS(p) & 10 & 6.6 \\
SMC [26] & DIS(p) & 12 & 4.6 \\
SLAC/E143 [31] & DIS(p) & 28 & 23.6 \\
SLAC/E155 [33] & DIS(p) & 24 & 22.3 \\
HERMES [22] & DIS(p) & 37 & 18.2 \\
COMPASS'10 [27] & DIS(p) & 15 & 11.1 \\
COMPASS'16 [28] & DIS(p) & 51 & 31.2 \\
CLAS/EG1b [16] & DIS(p) & 166 & 91.0 \\
SMC [26] & DIS(d) & 12 & 17.2 \\
SLAC/E143 [31] & DIS(d) & 28 & 41.4 \\
SLAC/E155 [34] & DIS(d) & 24 & 17.9 \\
HERMES [22] & DIS(d) & 37 & 35.7 \\
COMPASS [29] & DIS(d) & 43 & 29.8 \\
CLAS/EG1b [17] & DIS(d) & 158 & 136.2 \\
SLAC/E142 [30] & DIS(n) & 8 & 5.8 \\
SLAC/E154 [32] & DIS(n) & 17 & 5.4 \\
HERMES [35] & DIS(n) & 9 & 2.6 \\
JLab-Hall A [36] & DIS(n) & 3 & 1.4 \\
TOTAL: & & $\mathbf{6 8 2}$ & $\mathbf{5 0 1 . 6}$ \\
\hline \hline
\end{tabular}

In order to study the effects on the polarized PDFs on the deviation of $a_{8}$ from its $S U(3)$ symmetric value, we have performed the following fits:

(a) Fit A: The data is fitted using the $S U(3)$ symmetric value 0.586 for $a_{8}$ (7).

(b) Fit B: The data is fitted using $a_{8}=0.46$. This value corresponds to the maximal reduction of $a_{8}$ presented in the literature and is the value predicted in one of the models on $S U(3)$-breaking effects [13].

(c) Fit C: The data is fitted using $a_{8}$ as a free parameter.

Since the isospin $S U(2)$ symmetry is considered as almost exact, we have used the very precisely measured value $g_{A}=1.270$ (see Eq. (6)).
The numerical results of our NLO QCD Fit A to the present world $g_{1}$ data set are presented in Tables I-III.

In Table I the $g_{1}$ data sets used in our analysis are listed and the corresponding values of $\chi^{2}$ obtained from the best fit to the data are presented. As seen from Table I, a good description of the data is achieved: $\chi^{2} /$ d.o.f. $=0.759$ for 682 experimental points using 21 free parameters (11 for the PDFs and 10 for the higher twist corrections). The final proton and deuteron CLAS/EG1b data are well consistent with the previous world data set.

The values of the parameters attached to the input polarized PDFs obtained from the best fit to the data are presented in Table II. The errors used in the fit are quadratic combinations of the statistical and point-to-point systematic errors. As seen from Table II, the parameters describing the polarized strange quark density are well determined.

The extracted polarized NLO PDFs are plotted in Fig. 1 for $Q^{2}=2.5 \mathrm{GeV}^{2}$ and compared to those obtained by the groups ([3-5]). As seen from Fig. 1, our strange quark density is negative and consistent with that obtained in the previous analyses in which for $a_{8}$ its $S U(3)$ value was used. It is also seen that as a function of $x$, the shape of the JAM'15 strange quark density is harder than those obtained from the other groups. Note that contrary to the other groups the JAM Collaboration has used in his analysis [4] an alternative approach, based on a new iterative Monte Carlo fitting technique.

Note that the results for the polarized PDFs presented in the Table I and Fig. 1 correspond to Fit A using $\gamma_{s_{+}}=0$ in the input parametrization for the polarized strange quark density (5). As was shown in [6] the precision of the data available at that time was enough in order to determine well the parameter $\gamma_{s_{+}}$. This parameter is important in principle because it allows changing in sign behavior for the polarized strange quark density. So, we have repeated Fit A using $\gamma_{s_{+}}$as a free parameter, and obtained for it:

TABLE II. The parameters of the NLO input polarized PDFs at $Q^{2}=1 \mathrm{GeV}^{2}$ obtained from the best Fit A to the data. The errors shown are total (statistical and systematic). The parameters marked by (*) are fixed.

\begin{tabular}{lccccc}
\hline \hline flavor & $\mathrm{A}$ & $\alpha$ & $\beta$ & $\epsilon$ & $\gamma$ \\
\hline$u+\bar{u}$ & $1.9456^{*}$ & $0.8947 \pm 0.1658$ & $3.1975 \pm 0.1097$ & $-1.3766 \pm 0.8079$ & $5.2073 \pm 2.4380$ \\
$d+\bar{d}$ & $-0.5062^{*}$ & $0.5821 \pm 0.1256$ & $4.2776 \pm 0.3998$ & 0 & $5.0596 \pm 4.4773$ \\
$s+\bar{s}$ & $-0.2524 \pm 0.1663$ & $0.5624 \pm 0.1661$ & $9.0^{*}$ & 0 & 0 \\
$\mathrm{G}$ & $30.241 \pm 13.384$ & $2.9128 \pm 0.7419$ & $5.0^{*}$ & 0 & 0 \\
\hline \hline
\end{tabular}

TABLE III. Sensitivity of the first moments of the polarized parton densities to $S U(3)$ symmetry flavor symmetry breaking $\left(Q^{2}=2.5 \mathrm{GeV}^{2}\right)$. The $S U(3)$ value $3 \mathrm{~F}-D=0.586$.

\begin{tabular}{lcccccr}
\hline \hline$a_{8}$ & $\chi^{2} /$ d.o.f. & $(\Delta u+\Delta \bar{u})$ & $(\Delta d+\Delta \bar{d})$ & $(\Delta s+\Delta \bar{s})$ & $\Delta \Sigma$ & $\Delta G$ \\
\hline 3F-D (Fit A) & 0.759 & $0.815 \pm 0.025$ & $-0.455 \pm 0.030$ & $-0.113 \pm 0.020$ & $0.247 \pm 0.044$ & $0.328 \pm 0.161$ \\
0.46 (Fit B) & 0.759 & $0.805 \pm 0.024$ & $-0.465 \pm 0.029$ & $-0.060 \pm 0.016$ & $0.280 \pm 0.041$ & $0.331 \pm 0.160$ \\
\hline \hline
\end{tabular}



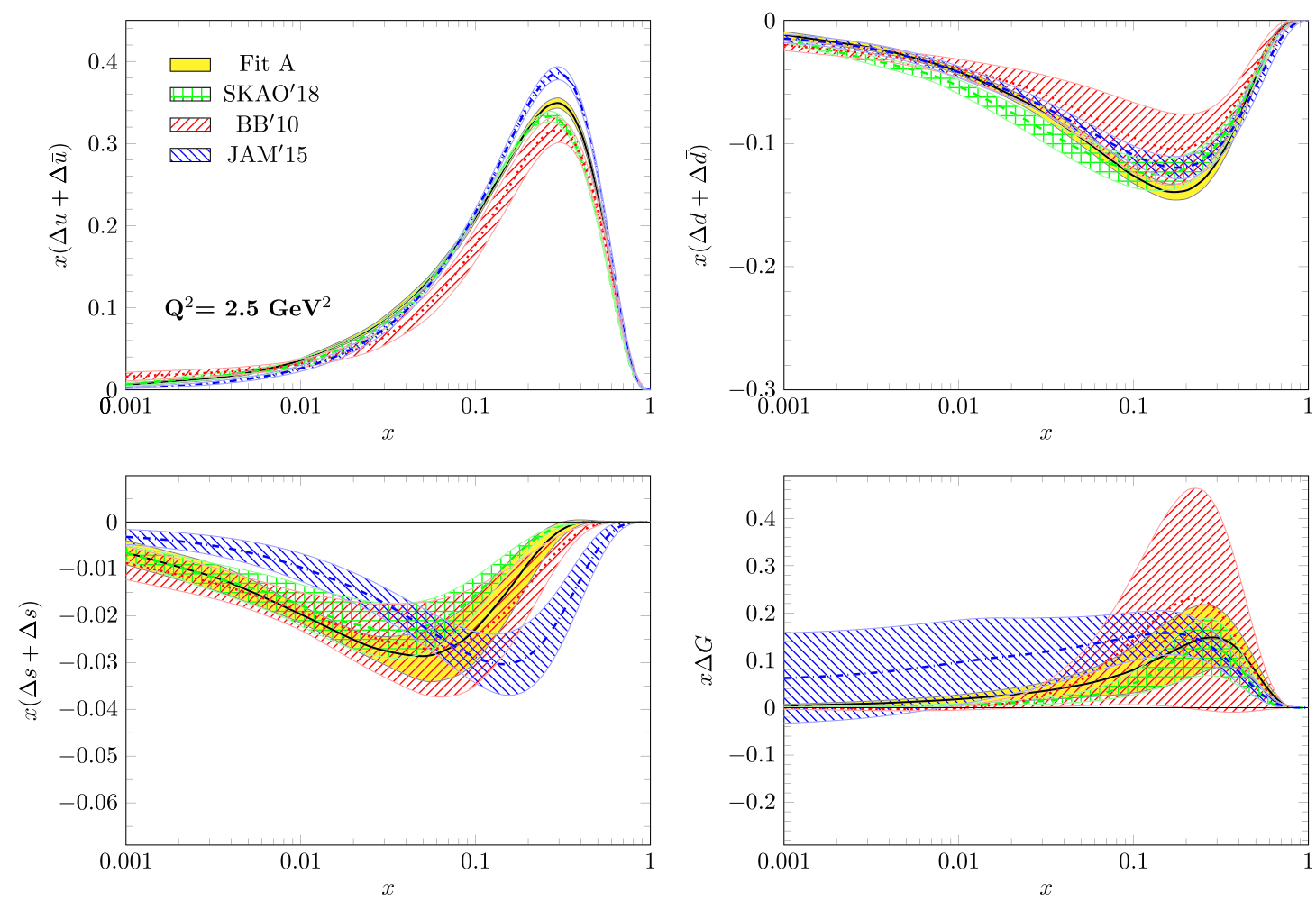

FIG. 1. Our NLO polarized PDFs obtained from Fit $\mathrm{A}\left(\gamma_{s_{+}}=0\right)$ compared to those of BB'10 [3], JAM'15 [4], and SKAO'18 [5].

$\gamma_{s_{+}}=-2.859 \pm 0.552$. The values obtained for $\chi^{2} /$ d.o.f. and the first moments of the quark densities, are essentially the same as in the case $\gamma_{s_{+}}=0$. So, including in the data fit one more parameter does not improve the description of the data. Although the first moment of $(\Delta s+\Delta \bar{s})(x)$ corresponding to $\left(\gamma_{s_{+}} \neq 0\right)$ is equal to that with $\left(\gamma_{s_{+}}=0\right)$, the behavior of the strange quark density is slightly different (see Fig. 2).

It is important to note that the strange quark density is negative for small values of $x$ and only changes sign in the

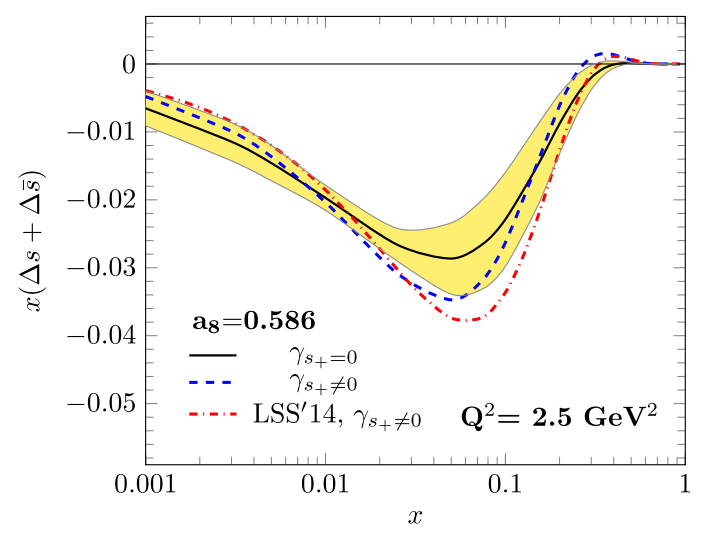

FIG. 2. Comparison between the strange quark densities obtained in the Fit A with $\gamma_{s_{+}}=0$ and $\gamma_{s_{+}} \neq 0$ (see the text). The LSS'14 strange quark density [6] $\left(\gamma_{s_{+}} \neq 0\right)$ is also shown. region $0.2<x<0.35$ (the precise point depending on the value of $Q^{2}$ ). Beyond this crossover point it is exceedingly small, compatible with zero (see Fig. 2). As seen from Fig. 2 it is consistent with the LSS' 14 strange quark density [6]. The difference in the large $x$ region is due to the different behavior of the unpolarized strange quark densities used in the positivity constraints, the MRST'02 set in [6] and MMHT'14 in this analysis.

Let us comment now the results of Fit B when for the nonsinglet axial charge $a_{8}$ instead of its $S U(3)$ symmetric value 0.586 , the value 0.46 have been used in the fit. In order to demonstrate the sensitivity of the parton densities to the $S U(3)$ breaking we present them (Fig. 3) and the values of their first moments in Table III, and compare them with those obtained in the Fit A. One can see from Table III that the values of $\chi^{2} /$ d.o.f. for both the fits are the same, so that the present polarized inclusive DIS data cannot distinguish between these two values. Note that all results of Fit B presented in the paper correspond to the parameter $\gamma_{s_{+}}=0$. The usage of $\gamma_{s_{+}}$as a free parameter does not essentially change the results and conclusions.

Contrary to the rest of the parton densities, which are essentially those determined by the $S U(3)$ analysis of the data, the polarized strange quark density changes significantly when flavor $S U(3)$ symmetry is broken (see Fig. 3 and Table III). Compared to the $S U(3)$ case the shape of the strange quark density is almost the same, but its magnitude is approximately halved. It is important to note that with the 

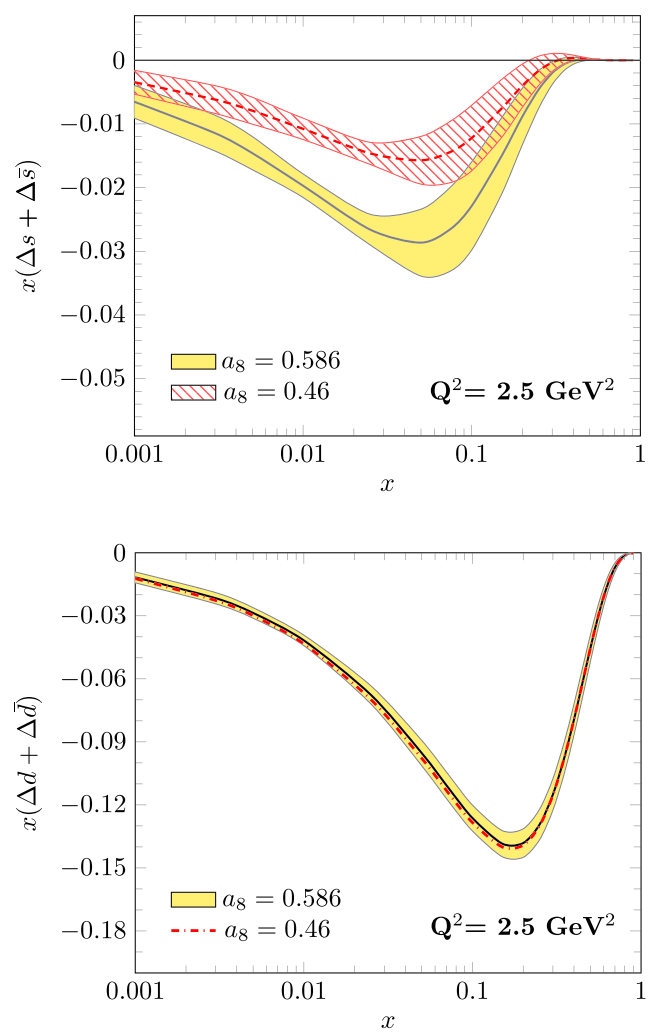

FIG. 3. Comparison between the polarized strange quark densities (top) and $(\Delta d+\Delta \bar{d})$ densities (bottom) obtained in the Fit A using for $a_{8}$ its $S U(3)$ value and Fit B $\left(a_{8}=0.46\right)$.

inclusion of much new data the strange quark density and its first moment remain significantly negative, in agreement with the result obtained by LSS group from the analysis of the polarized inclusive DIS data available at that time (see the footnote [27] in [6]).

As seen from Table III, as a result of the reduction of the $S U$ (3) value of $a_{8}$ by $22 \%$, causes the first moment of the singlet quark density, $\Delta \Sigma$ (the spin of the nucleon carried by the quarks), to increase by $13 \%$.

In Fig. 3, in addition to the polarized strange quark density, we show the fit $\mathrm{B}(\Delta d+\Delta \bar{d})$ parton density as an illustration that the rest of the polarized parton densities are almost identical to those obtained in the $S U(3)$ Fit A.

As usual the polarized gluon density is extracted from the inclusive DIS data with a larger uncertainty than that for the other densities. Nevertheless, we would like to mention that the value of the truncated first moment of our gluon density, $\int_{0.05}^{1} d x \Delta G(x)=0.28 \pm 0.12$ at $Q^{2}=10 \mathrm{GeV}^{2}$, is well consistent with that, $0.20 \pm 0.06$, determined from a global QCD analysis of the polarized parton densities [37] including the high-statistics RHIC data on the double-spin asymmetries for inclusive jet and $\pi^{0}$ production [38].

Finally, we will briefly mention our results for the fit to the data using $a_{8}$ as a free parameter (Fit C). For $\chi^{2} /$ d.o.f. we find the value 0.760 (practically the same value as for the Fits A and B, 0.759), and for $a_{8}=0.322 \pm 0.018$,

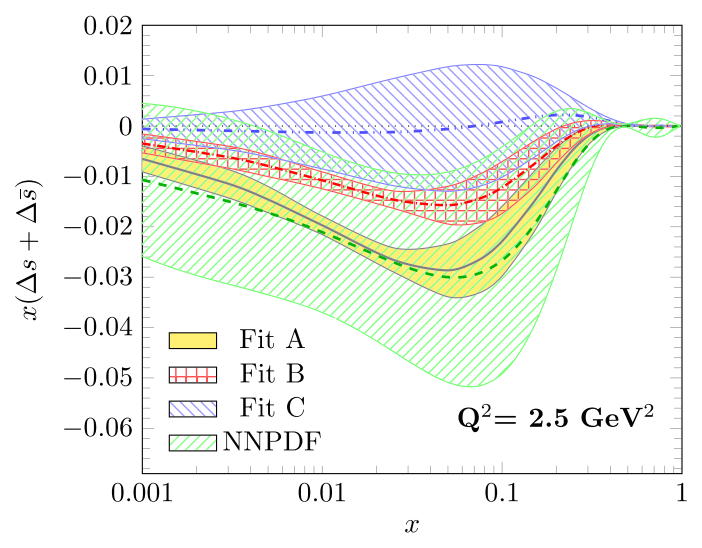

FIG. 4. Comparison between the strange quark densities obtained from Fits A, B, C, and NNPDFpol1.0 [14].

which implies a $45 \%$ violation of the $S U(3)$ flavor symmetry. However, such a strong violation of the $S U$ (3) flavor symmetry is in a big contradiction with the data on hyperon $\beta$ decays [11]. The strange quark density is presented in Fig. 4 and compared to those obtained in Fit A and Fit B. As seen from Fig. 4, it is consistent with zero for all $x$ values, and its uncertainty is larger than that obtained in Fit A and fit B.

In Fig. 4 is also shown the strange quark density (NNPDFpol1.0) obtained in the QCD analysis of the NNPDF Collaboration [14] of the inclusive DIS data, in which the flavor $S U(3)$ symmetry has been broken in another way. For the nonsinglet charge $a_{8}$ has been used the value $a_{8}=0.585 \pm 0.176$, i.e., for the uncertainty has been used a value approximately six times bigger than the value 0.03 obtained from the phenomenological analysis of the data on hyperon $\beta$ decays. This choice of the uncertainty actually means that the $S U(3)$ symmetry is up to $30 \%$ broken. As a result, the NNPDF central value for the strange quark density is close to ours (Fit A), however, their error band is much larger than that obtained in our Fit A, which is demonstrated in Fig. 4. (Note that our uncertainty for the Fit A strange quark density is close to those obtained in the fits using the value 0.03 for the uncertainty of $a_{8}$, see Fig. 1.)

We would like to mention also that in this analysis a $W^{2}$ cut of the data has been used in order to minimize the higher twist effects. However, such a cut of the data in the polarized case, results in the loss of information from the already much smaller amount of data than in the unpolarized case. Note that the uncertainty for the strange quark density is slightly improved in the small $\mathrm{x}$ region, $x<0.001$ from a combined analysis of the same collaboration [39], where to the polarized inclusive DIS data the polarized hadron collider data for inclusive jet and $\mathrm{W}$ boson production from the STAR and PHENIX experiments at RHIC have been added.

The JAM Collaboration, based on a global QCD analysis of polarized inclusive and semi-inclusive deep-inelastic 


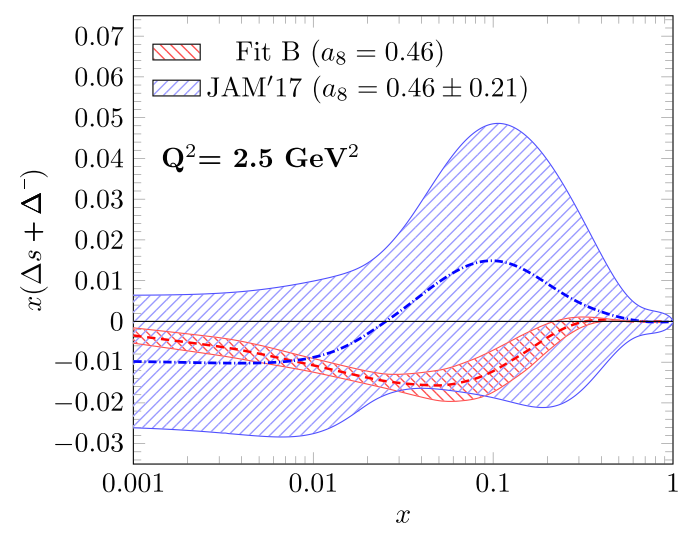

FIG. 5. Comparison between the strange quark densities obtained in the Fit B $\left(a_{8}=0.46\right)$ and JAM'17 [40].

scattering and single-inclusive $e^{+} e^{-}$annihilation data using $a_{8}$ as a free parameter [40], found the value 0.46 which we used in our Fit B. Thus in Fig. 5 we compare the polarized strange quark density obtained from fit B with the JAM'17 result. In the JAM'17 analysis the polarized parton densities and the fragmentation functions have been simultaneously extracted from the data, but the very precise JLab inclusive DIS data were not included in the fit. Also, the fragmentation functions are mainly fixed from the semiinclusive data on the longitudinal double spin asymmetries, which are much less precise than the unpolarized semiinclusive data on hadron multiplicities, the best source for their precise determination. As a result, the uncertainties for the polarized parton densities are much larger, especially for the strange quark density, than those obtained from the inclusive DIS data. As seen from Fig. 5, our Fit B polarized strange quark density together with its error band, entirely lies within the large error band of the JAM' 17 strange quark density.

Interestingly, the JAM' 17 value 0.46 for $a_{8}$ agrees with the value predicted in [13]. However, bearing in mind the large error, $45 \%$ of the magnitude of $a_{8}$, we are still far from really fixing the magnitude of the breakdown of the $S U(3)$ flavor symmetry from the polarized DIS and SIDIS data.

We would like to stress once more that in order to obtain reliable values for the polarized individual quark and antiquark densities, as well as for $a_{8}$, using SIDIS data, it is crucial that the FFs to be well determined. In this context it should be noted that the extracted pion and kaon FFs in the JAM'17 analysis are not consistent with those obtained from the global analysis [41], where the unpolarized SIDIS data on hadron multiplicities were used.

As was mentioned above [see Eq. (4)], we have taken into account the higher twist corrections $h^{N}\left(x_{i}\right) / Q^{2}$ to the spin structure functions in our fits to DIS data, treating the $h^{N}\left(x_{i}\right)$ as are free parameters. The values of the HT corrections $h^{p}\left(x_{i}\right)$ and $h^{n}\left(x_{i}\right)$ for the proton and neutron targets extracted from the data in this analysis are presented in Fig. 6. For the deuteron target the relation $h^{d}\left(x_{i}\right)=$

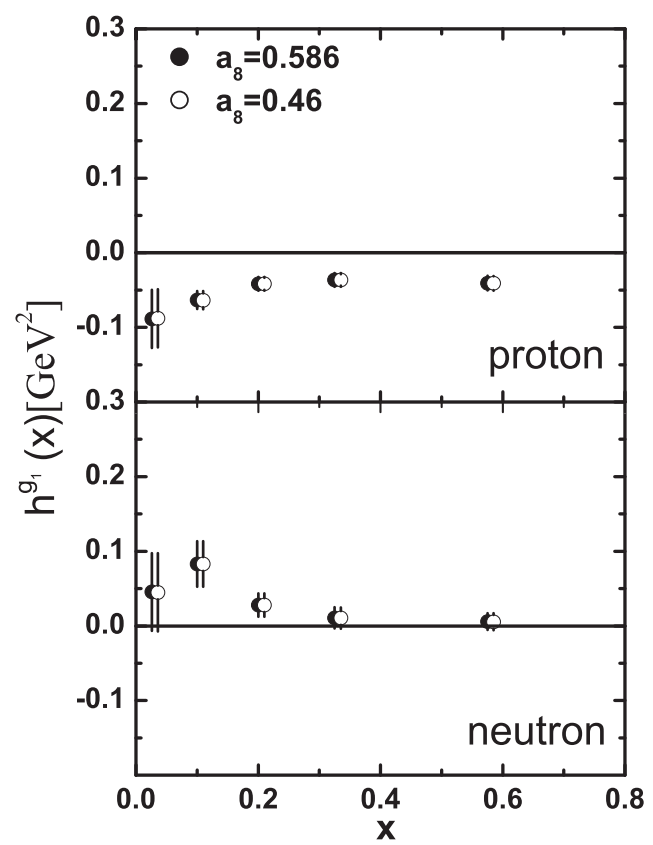

FIG. 6. Comparison between higher twist corrections corresponding to the values of $a_{8} 0.586$ and 0.46 .

$0.925\left[h^{p}\left(x_{i}\right)+h^{n}\left(x_{i}\right)\right] / 2$ has been used, where 0.925 is the value of the polarization factor $D$.

As seen from Fig. 6, the extracted from the data higher twist corrections are not sensitive to the value of nonsinglet axial charge $a_{8}$.

\section{SUMMARY}

We have presented a NLO QCD analysis of the present world inclusive DIS data on the nucleon spin structure functions $g_{1}^{N}$, in which the sensitivity of the polarized strange quark density to flavor $S U(3)$ symmetry breaking, i.e., to the deviation of the value of the nonsinglet axial charge $a_{8}$ from its $S U(3)$ symmetric value 0.586 , has been studied. Three fits to the data corresponding to different values of $a_{8}$ were performed using: (A) its $S U(3)$ symmetric value 0.586 , (B) the value 0.46 i.e., the maximal reduction of $a_{8}$ presented in the literature and obtained in a theoretical model, and (C) $a_{8}$ taken as a free parameter to be obtained from the best fit to the data.

It was shown that contrary to the rest of the parton densities, which hardly change from their $S U(3)$ analysis values, the polarized strange quark density changes significantly when flavor $S U(3)$ symmetry is broken. When $a_{8}=0.46$, the strange quark density and its first moment still remain significantly negative. Compared to the $S U(3)$ case, the shape is almost the same, but its magnitude is approximately halved. Using $a_{8}$ as a free parameter we obtain $a_{8}=0.322 \pm 0.018$ and in this case the strange quark density is consistent with zero. The above value for $a_{8}$ implies a $45 \%$ violation of $S U(3)$ flavor symmetry 
which is unlikely to be consistent with the data on hyperon $\beta$ decays.

An important feature of all the fits is that the data are well described and the value of $\chi^{2} /$ d.o.f. is practically the same for all three types of fit, which means that the inclusive polarized DIS data alone cannot distinguish between the different strange quark densities discussed above.

To improve the sensitivity to $S U(3)$ breaking and to the polarized strange quark density, at the very least, polarized semi-inclusive DIS data have to be involved, and such attempts have been made in the past ([7-10]) and very recently [40]. However, their success depends on how reliably the pion and kaon fragmentation functions used in the analysis are determined. Bearing in mind that the very precise HERMES and COMPASS experimental data on the pion and kaon multiplicities are inconsistent [42], it seems that the carrying out of such an analysis is, at present, not so easy. In the long run data from an Electron-Ion Collider, with a much larger range of $Q^{2}$, will help to constraint much better the strange quark polarization in the nucleon, but it is essential that any such results should correspond to the extracted value for $a_{8}$ being consistent with the data on hyperon $\beta$ decays.

\section{ACKNOWLEDGMENTS}

E. Leader and D. Stamenov are grateful to W. Melnitchouk for providing us with the JAM'15 and JAM'17 polarized PDFs, as well as for the useful discussion. A. K. appreciates A. Vogt and H. Abdolmaleki for discussions and guidance. This research was supported by Semnan University.
[1] E. Leader, A. V. Sidorov, and D. B. Stamenov, Phys. Rev. D 75, 074027 (2007).

[2] V. Y. Alexakhin et al. (COMPASS Collaboration), Phys. Lett. B 647, 8 (2007); M. Hirai and S. Kumano (Asymmetry Analysis Collaboration), Nucl. Phys. B813, 106 (2009).

[3] J. Blumlein and H. Böttcher, Nucl. Phys. B841, 205 (2010).

[4] N. Sato, W. Melnitchouk, S. E. Kuhn, J. J. Ethier, and A. Accardi, Phys. Rev. D 93, 074005 (2016).

[5] M. Salimi-Amiri, A. Khorramian, H. Abdolmaleki, and F. I. Olness, Phys. Rev. D 98, 056020 (2018).

[6] E. Leader, A. V. Sidorov, and D. B. Stamenov, Phys. Rev. D 91, 054017 (2015).

[7] D. de Florian, R. Sassot, M. Stratmann, and W. Vogelsang, Phys. Rev. D 80, 034030 (2009).

[8] E. Leader, A. V. Sidorov, and D. B. Stamenov, Phys. Rev. D 82, 114018 (2010).

[9] F. Arbabifar, A. N. Khorramian, and M. Soleymaninia, Phys. Rev. D 89, 034006 (2014).

[10] E. Leader, A. V. Sidorov, and D. B. Stamenov, Phys. Rev. D 84, 014002 (2011).

[11] N. Cabibbo, E. C. Swallow, and R. Winston, Annu. Rev. Nucl. Part. Sci. 53, 39 (2003).

[12] R. Flores-Mendieta, E. Jenkis, and A. Manohar, Phys. Rev. D 58, 094028 (1998); R. L. Jaffe and A. Manohar, Nucl. Phys. B337, 509 (1990); P. Ratcliffe, Phys. Rev. D 59, 014038 (1998).

[13] S. D. Bass and A. W. Thomas, Phys. Lett. B 684, 216 (2010).

[14] R. D. Ball, S. Forte, A. Guffanti, E. R. Nocera, G. Ridolfi, and J. Rojo (The NNPDF Collaboration), Nucl. Phys. B874, 36 (2013).

[15] E. Leader, A. V. Sidorov, and D. B. Stamenov, Phys. Lett. B 488, 283 (2000).

[16] R. Fersch et al. (CLAS Collaboration), Phys. Rev. C 96, 065208 (2017).
[17] N. Guler et al. (CLAS Collaboration), Phys. Rev. C 92, 055201 (2015).

[18] E. Leader, A. V. Sidorov, and D. B. Stamenov, Phys. Rev. D 58, 114028 (1998).

[19] A. Piccione and G. Ridolfi, Nucl. Phys. B513, 301 (1998); J. Blumlein and A. Tkabladze, Nucl. Phys. B553, 427 (1999); W. Detmold, Phys. Lett. B 632, 261 (2006).

[20] A. Vogt, Comput. Phys. Commun. 170, 65 (2005).

[21] J. Beringer et al. (Particle Data Group), Phys. Rev. D 86, 010001 (2012).

[22] A. Airapetian et al. (HERMES Collaboration), Phys. Rev. D 75, 012007 (2007).

[23] L. A. Harland-Lang, A. D. Martin, P. Motylinski, and R. S. Thorne, Eur. Phys. J. C 75, 204 (2015).

[24] E. Leader, A. V. Sidorov, and D. B. Stamenov, Phys. Rev. D 67, 074017 (2003).

[25] J. Ashman et al. (EMC Collaboration), Phys. Lett. B 206, 364 (1988); Nucl. Phys. B328, 1 (1989).

[26] B. Adeva et al. (SMC Collaboration), Phys. Rev. D 58, 112001 (1998).

[27] M. G. Alekseev et al. (COMPASS Collaboration), Phys. Lett. B 690, 466 (2010).

[28] C. Adolph et al. (COMPASS Collaboration), Phys. Lett. B 753, 18 (2016).

[29] C. Adolph et al. (COMPASS Collaboration), Phys. Lett. B 769, 34 (2017).

[30] P. L. Anthony et al. (SLAC E142 Collaboration), Phys. Rev. D 54, 6620 (1996).

[31] K. Abe et al. (SLAC E143 Collaboration), Phys. Rev. D 58, 112003 (1998).

[32] K. Abe et al. (SLAC/E154 Collaboration), Phys. Lett. B 405, 180 (1997).

[33] P. L. Anthony et al. (SLAC E155 Collaboration), Phys. Lett. B 493, 19 (2000).

[34] P. L. Anthony et al. (SLAC E155 Collaboration), Phys. Lett. B 463, 339 (1999). 
[35] K. Ackerstaff et al. (HERMES Collaboration), Phys. Lett. B 404, 383 (1997).

[36] X. Zheng et al. (JLab/Hall A Collaborations), Phys. Rev. Lett. 92, 012004 (2004); Phys. Rev. C 70, 065207 (2004).

[37] D. de Florian, R. Sassot, M. Stratmann, and W. Vogelsang, Phys. Rev. Lett. 113, 012001 (2014).

[38] P. Djawotho (STAR Collaboration), Nuovo Cimento C 036, 35 (2013); A. Adare et al. (Phenix Collaboration), Phys. Rev. D 90, 012007 (2014).
[39] E. R. Nocera, R. D. Ball, S. Forte, G. Ridolfi, and J. Rojo, Nucl. Phys. B887, 276 (2014).

[40] J. J. Ethier, N. Sato, and W. Melnitchouk, Phys. Rev. Lett. 119, 132001 (2017).

[41] D. de Florian, R. Sassot, M. Epele, R. J. Hernndez-Pinto, and M. Stratmann, Phys. Rev. D 91, 014035 (2015); 95, 094019 (2017).

[42] C. Adolph et al. (COMPASS Collaboration), Phys. Lett. B 764, 1 (2017); 767, 133 (2017). 